\title{
Tecnologias Digitais e o Desenvolvimento da Cognição Numérica: possibilidades para o ensino da Matemática
}

\section{Digital Technologies and the Development of the Numerical Cognition: possibilities for the Mathematics teaching}

\author{
Patrícia Ferreira Concato de Souza (patricia_concato@hotmail.com) \\ Secretaria de Educação, Prefeitura Municipal de Cornélio Procópio \\ Universidade Estadual do Norte do Paraná - campus de Cornélio Procópio (UENP / PPGEN) \\ João Coelho Neto (joaocoelho@uenp.edu.br) \\ Universidade Estadual do Norte do Paraná - campus de Cornélio Procópio (UENP / PPGEN) \\ Marília Bazan Blanco (mariliabazan@uenp.edu.br) \\ Universidade Estadual do Norte do Paraná - campus de Cornélio Procópio (UENP /PPGEN)
}

Resumo: O presente artigo visa investigar, à luz da Revisão Sistemática de Literatura, quais são as Tecnologias Digitais utilizadas no ensino da Matemática na Educação Básica, a fim de identificar quais instrumentos tecnológicos são utilizados para auxiliar o desenvolvimento da Cognição Numérica. Como encaminhamentos metodológicos foram realizadas adaptações das etapas de uma Revisão Sistemática de Literatura, para a qual foram analisados periódicos entre 2008 a 2018, nos seguintes bancos de dados: Instituto Brasileiro de Informação em Ciência e Tecnologia e nos periódicos com estratos na área de Ensino A1 a B1, classificados no índice restrito - quadriênio 2013-2016. Como resultado, dos 3141 artigos analisados, observou-se que não foi encontrado nenhum artigo relacionado ao tema foco da pesquisa, identificando a necessidade de pesquisa nas áreas de Tecnologia e do Senso Numérico e Cognição Numérica.

Palavras-chave: Cognição Numérica; Senso Numérico; Tecnologia Digital.

\begin{abstract}
The present paper aims to investigate, in the light of the Systematic Literature Review, which Digital Technologies are used in Mathematics teaching in Basic Education, in order to identify which technological instruments have been used to assist in the development of Numerical Cognition. As a methodological referral, adaptations to the steps of a Systematic Literature Review were made, in which periodicals between 2008 and 2018 were analyzed, in the following databases: Brazilian Institute of Information in Science and Technology and in periodicals with strata in the area of Education A1 to B1, classified in the restricted index - quadrennium 2013-2016. As a result, of the 3141 papers analyzed, it was observed that no article was found related to the topic of research, identifying the need for research in the areas of Technology and Numerical Sense and Numerical Cognition.
\end{abstract}

Keywords: Numerical Cognition; Numerical Sense; Digital Technology. 


\section{INTRODUÇÃO}

De acordo com os Parâmetros Curriculares Nacionais (Brasil, 1997, p. 26) "a Matemática deve ser vista pelo aluno como um conhecimento que pode favorecer o desenvolvimento do seu raciocínio, de sua capacidade expressiva, de sua sensibilidade estética e de sua imaginação".

Os conhecimentos matemáticos já estão presentes desde o nascimento da criança e com poucos meses de idade conseguem distinguir quantidades e até mesmo realizar cálculos mais simples, uma vez que o cérebro humano possui características que o habilita a lidar com os números, as habilidades numéricas (COSENZA; GUERRA; 2011).

Bastos (2010) corrobora com a ideia dos autores supracitados ao afirmar que "nos humanos, a representação interna para quantidades numéricas se desenvolve no $1^{\circ}$ ano de vida, servindo de base, mais tarde, para aquisição de habilidades para o aprendizado dos símbolos numéricos e realização de cálculos” (BASTOS, 2010, p. 26).

Habilidades numéricas são definidas como "[...] repertórios que ocorrem em contextos que envolvem numerosidade, problemas aritméticos, estimativas, cálculos, etc." (LORENA, CASTRO-CANEGUIM, CARMO, 2013, p. 44). Desse modo, são capacidades fundamentais para a aprendizagem da Matemática, as quais envolvem a identificação de números, estimativas, percepção de símbolos numéricos, relação número e quantidade, resolução de cálculos e a compreensão das regras aritméticas. De acordo com Silva (2016, p. 16) "os estudos dessas habilidades têm como base o desenvolvimento da Cognição Numérica”.

A Cognição Numérica é definida por Santos et al. (2016, p. 64) como “[...] parte das neurociências que estuda as bases cognitivas, neurais e do desenvolvimento dos números e matemática".

A estrutura funcional da cognição numérica é constituída por habilidades primárias e secundárias, uma vez que a primeira se compõe pelo senso numérico, a subitização ${ }^{1}$ e a estimativa. Já as habilidades secundárias são compostas por processamento numérico, produção numérica, compreensão numérica e cálculo.

\footnotetext{
${ }^{1}$ Subitização ou Subitizing é definida como um processo automático para determinar a magnitude de um pequeno conjunto de itens (MOLINA, 2015, p.126).
} 
Para Geary (2004) ter um senso numérico pouco desenvolvido ocasiona dificuldades em aprendizagens posteriores como a contagem, operações aritméticas simples e cálculos, uma vez que a habilidade primária é uma estrutura fundamental para as aprendizagens posteriores.

O resultado do Sistema de Avaliação da Educação Básica (SAEB, 2017), avaliação que permite realizar um diagnóstico do desempenho escolar, constatou que apenas $15.52 \%$ dos alunos brasileiros até o quinto ano são proficientes na matemática, 33,12\% tem a aprendizagem básica e que $51,35 \%$ tem desempenho insuficiente na disciplina. Por meio dos resultados entende-se que muitas crianças apresentam dificuldade de aprendizagem.

Blanco et al. (2014) afirmam que:

É muito comum nos deparar com alunos desmotivados em aprender a Matemática, e as causas são as mais diversas: desconhecimento de sua importância no cotidiano, despreparo do professor, desinteresse por parte do aluno, fatores sociais ou a presença de alguma dificuldade de aprendizagem. (BLANCO et al., 2014, p. 3)

Sendo assim, é fundamental que o professor entenda como o seu aluno se apropria dos conteúdos trabalhados em sala de aula, para que o mesmo utilize estratégias que incentivem a criança a aprender a Matemática. Para Lara (2004, p.144) "o professor precisa perceber como o aluno sente-se motivado, entusiasmado e desafiado, assim como também ele mesmo deve ser autoconhecer para agir como um entusiasta em sala de aula".

Portanto, a partir da contextualização da pesquisa, o objetivo geral é identificar quais são os jogos eletrônicos e de que forma esses instrumentos tecnológicos estão sendo utilizados para auxiliar o desenvolvimento da Cognição Numérica à luz de uma Revisão Sistemática de Literatura. Para esse procedimento, teve-se como recorte temporal o intervalo de 2008 a 2018, com o intuito de quantificar e analisar os trabalhos que abordavam a temática de interesse, visando um mapeamento da área e viabilizando futuros trabalhos.

Utilizou-se, como fonte de dados para a pesquisa, trabalhos publicados no Banco de Dissertações do Instituto Brasileiro de Informação em Ciências e Tecnologia (IBCT), na opção "Busca Avançada", com as palavras-chave "Cognição Numérica" ou "Senso Numérico", bem como em revistas com escopo na área de Informática na Educação, encontradas no índice restrito (A1 a B1), na área de Ensino - Classificação de Periódicos Quadriênio 2013 a 2016. 
Assim, esse artigo foi dividido em quatro seções, sendo que a primeira contextualiza a temática; na segunda, os materiais e métodos utilizados são apresentados; na terceira, expõe-se os resultados e discussões e na quarta e última seção, tecem-se as considerações finais e os esboços de trabalhos futuros.

\section{Aporte Teórico}

\section{Desenvolvimento da Cognição Numérica}

O domínio da Matemática está associado ao desenvolvimento da cognição numérica, que envolve a "capacidade para representar quantidades nos âmbitos cognitivo e neural, por meio de seus sistemas inatos e adquiridos" (SANTOS, 2017, p.192)

A partir dessa definição, cognição numérica é composta por habilidades primárias e habilidades secundárias, uma vez que a primeira é uma habilidade inata do ser humano, o indivíduo não utiliza o princípio da contagem para reconhecer, comparar, identificar pequenas quantidades. Já as habilidades secundárias envolvem o conceito de número, a contagem, a compreensão dos símbolos numéricos, a aritmética e o cálculo, pois são construídas a partir do desenvolvimento das habilidades primárias. No entanto, as habilidades secundárias dependem da escolarização, pois incluem atividades mais complexas (GEARY, 2000)

Silva (2016), elaborou uma estrutura funcional da Cognição Numérica, o qual facilita a compreensão desse sistema: 


\section{Cognição Numérica}

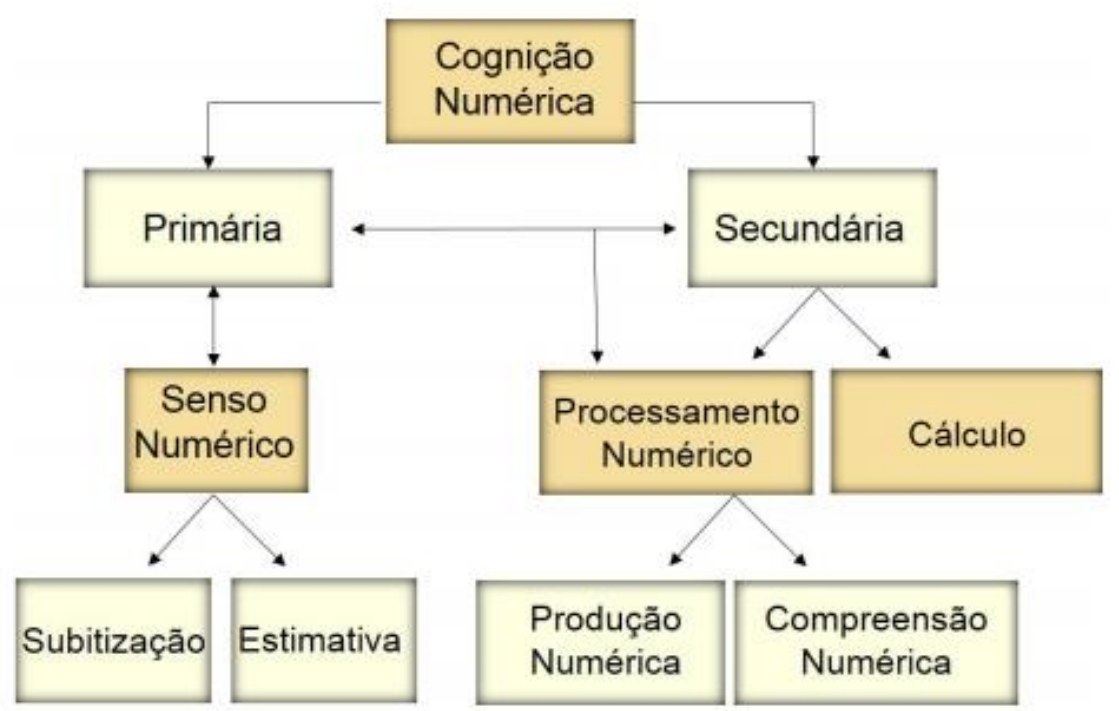

Fonte: Silva (2016, p. 19)

As pesquisas de Dehaene (1997), Corso e Dorneles (2010), Santos (2016), Molina et al. (2015) e Silva (2016) apontam que crianças com cognição numérica pouco desenvolvida podem apresentar dificuldades na matemática, uma vez que esta é a base para o desenvolvimento dos conteúdos posteriores.

O Senso Numérico é composto por duas capacidades, a subitização que está associada a "discriminação e reconhecimento de numerosidade até quatro" e pela estimativa, que é "julgamento aproximado de quantidades iguais ou superiores a cinco" (SILVA, 2016, p.19).

Ramos, Goodwin e Laudares $(2015$, p. 5) afirmam que:

[...] um senso numérico pouco desenvolvido pode se dever a uma representação e/ou processamento imaturo dos números que ocasiona defasagens na compreensão e flexibilidade no seu uso e acarreta problemas para o desenvolvimento de habilidades do tipo contagem, realização de operações, estimativas e cálculo mental.

Já as habilidades secundárias envolvem o processamento numérico, que está relacionado a compreensão e a produção numérica. A primeira permitirá que a criança relacione os símbolos numéricos as quantidades e segunda está associada a escrita, a leitura e a contagem. Essa capacidade é resultado do desenvolvimento das habilidades 
numéricas primárias, ou seja, o Senso Numérico (MC CLOSKEY, CARAMAZZA, BASILI, 1985).

Molina (2015, p. 126) explica as habilidades secundárias da seguinte maneira:

O processamento numérico se refere à compreensão do símbolo numérico associado às suas quantidades, capacidade essa que estaria ligada ao senso numérico e aos primeiros estágios de desenvolvimento das habilidades matemáticas em pré-escolares e a produção numérica que inclui escrita, leitura, e contagem numérica. Em contrapartida, a habilidades de cálculo se refere ao processamento dos símbolos ou palavras operacionais, à recuperação junto a memória de longo prazo de fatos aritméticos básicos.

Diante dos conceitos apresentados, compreende-se que domínio da Matemática é imprescindível para a vida em sociedade e que a mesma depende do desenvolvimento da Cognição Numérica. Desse modo, a presente pesquisa tem como objetivo identificar trabalhos que utilizam a tecnologia digital, com foco nos jogos eletrônicos educacionais, como um instrumento para auxiliar o desenvolvimento dessa capacidade.

\section{Tecnologias digitais como estratégia de Ensino}

A tecnologia digital está inserida fortemente na rotina dos alunos e, consequentemente, na sala de aula. Nesse sentido, Cardoso, Oliveira e Kato (2013, p. 1) afirmam que "[...] tornou-se parte indissociável do ofício do professor agregar metodologias que integram as mídias digitais ao seu cotidiano profissional". Para Coelho e Altoé (2008), as tecnologias digitais não são novidades no âmbito escolar e as mesmas ainda contribuem para a criação de novas estratégias de ensino e de aprendizagem.

Assim, o computador, o notebook, a internet e os dispositivos móveis, são as tecnologias digitais mais presentes nas instituições escolares. Dispositivos moveis são os aparelhos celulares e tablets, os quais estão em constante aperfeiçoamento. Higuchi (2011) explica que o diferencial dessa tecnologia é a mobilidade e portabilidade, além do que o indivíduo pode acessar diversas informações, assistir vídeos, realizar leituras a qualquer instante.

Entretanto, o computador é uma das tecnologias mais utilizadas e de fácil acesso nas instituições, visto que alguns estabelecimentos não possuem tablets, nem notebook e determinados estudantes ainda não possuem idade adequada para manusear os aparelhos celulares. 
$\mathrm{Na}$ concepção de Valente "o computador pode ser também para enriquecer ambientes de aprendizagem e auxiliar o aprendiz no processo de construção só seu conhecimento" (VALENTE, 1999, p. 12).

Utilizar esses recursos na sala de aula é mais do que ligar o computador e fazer uma simples pesquisa em sítios de busca: é possibilitar e sustentar atividades especiais, a fim de transformá-las em ações pedagógicas e em recursos que realmente somem importantes contribuições ao trabalho dos professores e à construção do conhecimento pelos alunos (COELHO NETO; ALTOÉ, 2009, p. 954)

Nessa perspectiva, o computador é visto como instrumento motivador da aprendizagem, uma vez que ele atrai a atenção e provoca o interesse em realizar atividades diferenciadas.

Teixeira e Coelho Neto (2016, p.02) afirmam que:

[...] a inserção do computador como instrumento colaborador no processo de ensino e aprendizagem é consideravelmente recente, em relação a outros meios de tecnologia educacional e tem se mostrado notável facilitador de procedimentos que antes levavam dispendioso tempo para serem executados.

O computador é um instrumento para o professor elaborar atividades, utilizar jogos eletrônicos, acessar ambientes virtuais de aprendizagem e software educativos. Tarouco et al. (2004) descrevem o computador como um recurso poderoso, o qual possibilita ao professor melhorar a sua prática pedagógica utilizando todos os recursos multimídia que a máquina oferece, "tais como jogos educacionais, vídeos, animações, gráficos, e outros materiais que possibilitem ao aluno aprender de forma prazerosa, cativante, divertida e motivadora" (TAROUCO et al., 2004, p. 1).

Destarte, Ramos (2009) aponta contribuições que a utilização do computador proporciona aos alunos:
a) oferece possibilidade de interação;
b) permite o acesso a informações;
c) desperta mais interesse das crianças pelas aulas;
d) possibilita a troca de informações;
e) amplia os espaços da sala de aula;
f) viabiliza a socialização de trabalhos através de outros meios;
g) prepara o aluno para o mercado de trabalho;
h) contribui com a inclusão digital dos alunos;
i) torna a aula mais interativa;

Com os apontamentos apresentado por Ramos (2009), compreende-se que o uso das tecnologias digitais no ensino contribui de forma peculiar para a aprendizagem, podendo 
ser um instrumento que auxilia crianças que possuem dificuldades no processo de aprendizagem.

Coelho Neto (2009) explica que as tecnologias digitais podem facilitar o processo de ensino e de aprendizagem, tornando-se um instrumento de ensino mais atraente, uma vez que a transmissão dos conteúdos não ocorrerá de maneira pronta e acabada e com o intuito da memorização dos conceitos, mas estimulará a capacidade criativa e exploratória dos alunos.

Ao utilizar tecnologias digitais em suas práticas pedagógicas, o docente oportunizará o reforço dos conteúdos trabalhados em sala, que facilitará a compreensão dos conceitos. "Neste instante de criação e elaboração, o aluno busca a superação das dificuldades e problemas que vão surgindo neste processo, assim estes permanecem em contato com diversos conhecimentos possíveis de serem assimilados (GENEROSO et al., 2013, p. 232).

Um mesmo jogo pode ser trabalhado diversas vezes, não com o objetivo de decorar o conteúdo, mas para que o aluno possa abstrair e entender as temáticas tratadas em sala de aula. Ao apresentar um novo assunto, essa tecnologia digital permitirá ao aluno buscar um conhecimento para resolução da situação proposta, assim o mesmo associará a importância real dos conteúdos para sua vida em sociedade (FERNANDES, 2011).

Desse modo, os jogos eletrônicos educacionais, como tecnologias digitais, têm objetivo de favorecer a aquisição dos conhecimentos e inovar as práticas pedagógicas do professor. Assim, a aprendizagem por meio das tecnologias digitais possibilita ao aluno sistematizar os conteúdos, criar estratégias para resolver situações problemas e a respeitar regras que são impostas pelos programas utilizados.

\section{Materiais e Métodos}

O presente trabalho se caracteriza como uma pesquisa qualitativa, pois de acordo com Gerhardt e Silveira (2009, p.32) essa abordagem de pesquisa "[...] preocupa-se, portanto, com aspectos da realidade que não podem ser quantificados, centrando-se na compreensão e explicação da dinâmica das relações sociais". Para a coleta dos dados, utilizou-se as etapas de uma Revisão Sistemática de Literatura, a fim de identificar, avaliar e interpretar todas as pesquisas que abordassem a temática (KITCHENHAM, 2004). 
Para que a Revisão Sistemática de Literatura fosse executada, a pesquisa percorreu adaptações das etapas definidas por Kitchenham (2004, p.12), elencadas da seguinte forma: "Identificação de pesquisa; Seleção de estudos; Avaliação da qualidade do estudo; Extração de dados e monitoramento do progresso; Síntese de dados”.

Seguindo as etapas propostas pela pesquisadora supracitada, a questão elencada para essa pesquisa foi: Quais as tecnologias digitais utilizadas para o desenvolvimento da Cognição Numérica?

Para seleção dos estudos, seguiu-se os seguintes critérios: seleção de artigos, dissertações e teses que apresentavam as palavras "cognição numérica" ou "senso numérico" no título do trabalho e que estivessem associadas a tecnologia digital.

$\mathrm{Na}$ terceira e quarta etapa foi realizada a leitura dos artigos, dissertações e teses que se enquadravam nos critérios de seleção, a fim de realizar a avaliação e a extração dos dados. E como síntese de dados, selecionou-se os trabalhos relevantes, que serão apresentados na próxima sessão.

Para responder essa questão, foram utilizadas como fonte de dados a Biblioteca Digital de Teses e Dissertações (BDTD) disponível no Instituto Brasileiro de Informação em Ciência e Tecnologia (IBICT), e as revistas com escopo na área de Ensino, encontradas no índice restrito (A1 a B1), na área de Ensino - Classificação de Periódicos Quadriênio 2013 a 2016, cujo escopo relaciona-se à temática Informática na Educação, que possuíssem o termo "Informática" ou "Tecnologia" no título.

A pesquisa foi efetuada no segundo semestre de 2018, podendo haver alterações em pesquisas futuras. A partir dos critérios de inclusão da revisão, selecionou-se as seguintes revistas: Revista Brasileira Tecnologia (A2); Revista Brasileira de Informática na Educação (B1); Revista Informática na Educação (B1); Revista Ensino de Ciências e Tecnologia (B1); Revista Novas Tecnologias na Educação - RENOTE (B1); Revista Ciências e Tecnologia (B1); Revista Tecnologia e Sociedade (B1); Revista Tecnologia na Educação (B1) e Revista Tear (B1).

\section{Resultados e Discussões}

Na primeira fase da pesquisa, foram mapeados os artigos publicados nas principais revistas em língua portuguesa, qualificadas na área de Ensino como A1 a B1 (índice 
restrito) na plataforma WebQualis - classificação de periódicos quadriênio 2013-2016, cujo escopo dessas enquadravam-se na temática de Informática na Educação, no intervalo dos últimos dez anos (2008 a 2018), sendo a busca realizada nos meses de abril a julho de 2018, podendo ter alterações em buscas futuras, visto as mesmas terem novas edições. Houve uma leitura detalhada dos títulos dos artigos listados pelo sumário das edições, a fim de eliminar os trabalhos que não abordavam a temática da informática/tecnologia envolvendo Senso Numérico e/ou Cognição Numérica, e conjuntamente foram analisados os trabalhos que tratavam da temática para leitura completa do manuscrito.

O Quadro 1 apresenta uma síntese dos resultados da pesquisa realizada em algumas revistas da plataforma WebQualis nos últimos dez anos, entretanto, não foi encontrado nenhum trabalho que abordasse a temática:

Quadro 1: Mapeamento dos artigos nos periódicos

\begin{tabular}{|c|c|c|c|c|c|}
\hline Periódico & Qualis & ISSN & $\begin{array}{c}\text { Período de } \\
\text { Levantamento }\end{array}$ & $\begin{array}{c}\text { Total de Artigos } \\
\text { Pesquisados }\end{array}$ & $\begin{array}{c}\text { Total de artigos } \\
\text { que abordavam } \\
\text { a temática }\end{array}$ \\
\hline $\begin{array}{c}\text { Revista Brasileira } \\
\text { de Ensino } \\
\text { Ciências e } \\
\text { Tecnologia }\end{array}$ & A2 & $1982-873 X$ & $2008-2018$ & 392 & 0 \\
\hline $\begin{array}{c}\text { Ensino de } \\
\text { Ciências e } \\
\text { Tecnologia }\end{array}$ & B1 & $2237-4450$ & $2011-2018$ & 119 & 0 \\
\hline $\begin{array}{c}\text { RENOTE } \\
\text { RENormática na } \\
\text { Educação }\end{array}$ & B1 & $1982-1654$ & $2008-2018$ & 290 & 0 \\
\hline $\begin{array}{c}\text { Revista } \\
\text { Tecnologia e } \\
\text { Sociedade }\end{array}$ & B1 & $1984-3526$ & $2008-2018$ & 294 & 0 \\
\hline $\begin{array}{c}\text { Revista } \\
\text { Tecnologia na } \\
\text { Educação }\end{array}$ & B1 & $1984-4751$ & $2009-2018$ & 452 & 0 \\
\hline $\begin{array}{c}\text { Revista Ciência e } \\
\text { Tecnologia }\end{array}$ & B1 & $1851-7587$ & $2008-2018$ & 146 & 0 \\
\hline
\end{tabular}


Vol. 2, n. 2. Mai. /Ago. 2019

\begin{tabular}{|l|l|l|l|l|l|} 
Revista Tear & B1 & $2238-8079$ & $2012-2017$ & 206 & 0 \\
\hline
\end{tabular}

Fonte: Os autores

Em um segundo momento da pesquisa, na Biblioteca Digital de Teses e Dissertações (BDTD) do Instituto Brasileiro de Informação em Ciência e Tecnologia (IBICT), retornaram 9 trabalhos com a palavra-chave "Cognição Numérica", porém, apenas 5 estavam citados no título da pesquisa. No Quadro 2 são apresentados os trabalhos encontrados na busca na BDTD.

Quadro 2 - Teses e Dissertações Biblioteca Digital Brasileira de Teses e Dissertações do Instituto Brasileiro de Informação em Ciência e Tecnologia (IBCT)

\begin{tabular}{|c|c|c|c|}
\hline TÍTULO & AUTOR & PROGRAMA & ANO \\
\hline $\begin{array}{c}\text { O viés da cognição numérica } \\
\text { e práticas de controladoria: } \\
\text { um estudo quase? } \\
\text { Experimental. }\end{array}$ & $\begin{array}{l}\text { Marcelino, } \\
\text { Carolina Venturini. }\end{array}$ & $\begin{array}{l}\text { Mestrado em Contabilidade da } \\
\text { Faculdade de Ciências Contábeis } \\
\text { da Universidade Federal da Bahia }\end{array}$ & 2011 \\
\hline $\begin{array}{l}\text { O efeito do treino musical } \\
\text { sobre a capacidade da } \\
\text { memória operacional e da } \\
\text { cognição numérica de } \\
\text { crianças com discalculia do } \\
\text { desenvolvimento. }\end{array}$ & $\begin{array}{l}\text { Ribeiro, Fabiana } \\
\text { Silva }\end{array}$ & $\begin{array}{l}\text { Mestrado em psicologia do } \\
\text { Desenvolvimento e } \\
\text { Aprendizagem, Universidade } \\
\text { Estadual Paulista "Júlio de } \\
\text { Mesquita Filho" - Bauru, SP }\end{array}$ & 2013 \\
\hline $\begin{array}{c}\text { Implicações dos } \\
\text { polimorfismos VAL158MET } \\
\text { da CATECOL-O- } \\
\text { METILTRANSFERASE em } \\
\text { diferentes aspectos da } \\
\text { cognição numérica }\end{array}$ & $\begin{array}{l}\text { Costa, Annelise } \\
\text { Julio }\end{array}$ & $\begin{array}{c}\text { Mestrado em Neurociências, } \\
\text { Universidade Federal de Minas } \\
\text { Gerais }\end{array}$ & 2014 \\
\hline $\begin{array}{l}\text { Treino musical como } \\
\text { proposta para a estimulação } \\
\text { da cognição numérica em } \\
\text { crianças de idade escolar. }\end{array}$ & $\begin{array}{c}\text { Rodriguez, Indira } \\
\text { Arias }\end{array}$ & $\begin{array}{l}\text { Mestrado em Psicologia do } \\
\text { Desenvolvimento e } \\
\text { Aprendizagem, Universidade } \\
\text { Estadual Paulista "Júlio de } \\
\text { Mesquita Filho" - Bauru, SP }\end{array}$ & 2015 \\
\hline
\end{tabular}


Os efeitos do treino musical sobre a cognição numérica e a memória operacional: um estudo prospectivo em crianças pré-escolares.
Silva, Eder Ricardo da.
Mestrado em Psicologia do

Desenvolvimento e Aprendizagem, Universidade Estadual Paulista "Júlio de Mesquita Filho" - Bauru, SP
2016

Fonte: Os autores

Ainda na Biblioteca Digital Brasileira de Teses e Dissertações do Instituto Brasileiro de Informação em Ciência e Tecnologia, retornaram 12 trabalhos com a palavra-chave "Senso Numérico" em busca avançada, porém apenas 1 (uma) pesquisa apresentava a temática no título, conforme descrito no Quadro 3:

Quadro 3 - Teses e Dissertações Biblioteca Digital Brasileira de Teses e Dissertações do Instituto Brasileiro de Informação em Ciência e Tecnologia (IBCT)

\begin{tabular}{|c|c|c|c|}
\hline TÍTULO & AUTOR & PROGRAMA & ANO \\
\hline $\begin{array}{l}\text { O senso numérico da } \\
\text { criança: formação e } \\
\text { características. }\end{array}$ & $\begin{array}{c}\text { Ferrari, } \\
\text { Alessandra Hissa }\end{array}$ & $\begin{array}{c}\text { Doutorado em Educação } \\
\text { Matemática, Universidade } \\
\text { Católica de São Paulo - São } \\
\text { Paulo }\end{array}$ & 2008 \\
\hline
\end{tabular}

Fonte: Os autores

Por meio da pesquisa nas principais revistas da plataforma WebQualis, e no IBICT não foi encontrada nenhuma pesquisa que tratasse a temática da Tecnologia Digital no desenvolvimento da Cognição Numérica e/ou Senso Numérico.

Marcelino (2011) investigou a cognição numérica em práticas atreladas ao orçamento, analisando se há interferência na manifestação desse fenômeno quando se consideram fatores como o envolvimento em práticas orçamentárias, idade e gênero. Diante do estudo, foi possível concluir que o nível de envolvimento em práticas orçamentárias não interfere no viés da cognição numérica.

Ribeiro (2013) descreve o estudo sobre os efeitos musicais no desenvolvimento da memória operacional e da cognição numérica, além de outras funções cognitivas como inteligência, funções executivas e comportamento emocional.

Costa (2014) investiga as influências do Polimorfismo Val 158met da Enzima catecol-o-metiltransferase (COMT) sobre a Cognição Numérica na aprendizagem 
Matemática, sendo um trabalho pioneiro na área das bases genéticas da ansiedade matemática.

Rodrigues (2015) destaca que o treinamento musical pode ser um instrumento de intervenção e estimulação neurocognitiva, especialmente na cognição numérica, com crianças em idade escolar. O objetivo consiste em caracterizar o perfil das crianças com dificuldade aritmética, em medidas cognição numérica (Senso Numérico, produção e compreensão numérica, cálculo) e de memória operacional em contraste com o desenvolvimento típico.

Silva (2016) considerou ainda que o treinamento musical contribuiu para modificar o processamento numérico, o senso numérico e a memória operacional verbal.

Ferrari (2008) investigou a formação do conceito numérico da criança. A pesquisa abordou duas perspectivas: “contexto teórico com abordagens científicas, a partir das análises teóricas de Piaget e as ideias do neurocientista Stanislas Dehaene; e a segunda na identificação de diferenças individuais de habilidades numéricas em crianças que iniciam a vida escolar por meio de atividades empíricas". Nesse sentido, a autora utilizou um estudo de caso descritivo-analítico, pois as descrições foram necessárias para que as intervenções fossem realizadas.

Diante da pesquisa realizada, por meio de ações adaptadas de uma Revisão Sistemática de Literatura, esperava-se identificar artigos, dissertações e teses, que apresentassem a Tecnologia Digital como instrumento auxiliador no desenvolvimento da Cognição Numérica, com foco nos jogos eletrônicos educacionais. Assim, os jogos eletrônicos voltados para o processo de ensino e de aprendizagem, deveriam ter como objetivo estimular as habilidades primárias, como o senso numérico, a subitização e a estimativa, e as habilidades secundárias, processamento numérico e os cálculos.

Portanto, foi possível constatar uma defasagem de pesquisas nessa área, uma vez que os trabalhos selecionados de acordo com os critérios de inclusão e exclusão não apresentavam práticas tecnológicas para o desenvolvimento da Cognição Numérica.

\section{Considerações Finais}

Por meio das leituras foi possível constatar que a Tecnologia Digital mais utilizada nas instituições escolares é o computador, sendo um instrumento motivador da 
aprendizagem, a qual proporciona ao aluno a construção do conhecimento, elaboração de estratégias para resolução de situações-problemas, estimulando a atenção e o interesse em realizar atividades matemáticas.

Nessa perspectiva, a Tecnologia Digital pode ser uma abordagem facilitadora no processo de ensino e de aprendizagem, visto que esta faz parte do cotidiano dos indivíduos. A partir das leituras realizadas, pode verificar que, nessa pesquisa, não houve indícios de pesquisas que discutem essa temática como um instrumento no desenvolvimento da Cognição Numérica, sendo uma limitação para essa pesquisa.

Com as informações citadas nos quadros, emergem conhecimentos para responder as seguintes questões: Quais as Tecnologias Digitais utilizadas para o desenvolvimento da Cognição Numérica? Após realizar a pesquisa no periódico da Coordenação de Aperfeiçoamento de Pessoal de Nível Superior (CAPES) e analisar três mil cento e quarenta e um artigos, foi possível identificar uma falta de pesquisas que abordassem a temática.

Nas teses e dissertações do IBCT, foram encontradas apenas seis pesquisas seguindo o critério de seleção, com as palavras-chave "Cognição Numérica" ou "Senso Numérico" presentes no título. Entretanto, as pesquisas estavam associadas as palavras-chave, mas não abordavam a Tecnologia Digital como auxiliador para o desenvolvimento dessa habilidade cognitiva para o âmbito educacional.

Dessa forma, o objetivo geral desse trabalho foi identificar quais os instrumentos tecnológicos estão sendo utilizados para auxiliar o desenvolvimento da Cognição Numérica à luz de uma Revisão Sistemática de Literatura. Assim, não foi possível perceber esse vínculo nessa pesquisa, pois não foram encontrados trabalhos com esta temática, evidenciando-se a carência de Tecnologias Digitais como instrumento auxiliador no desenvolvimento da Cognição Numérica.

\section{Referências bibliográficas}

BASTOS, José Alexandre. O Cérebro e a Matemática. 1. ed. São José do Rio Preto - SP: Edição do Autor

BLANCO, M. et. al. O Uso de Recursos Midiáticos no Atendimento de Crianças com Discalculia em Sala de Recursos de Escolas Estaduais do Norte do Estado do Paraná. In: 
Congresso Iberoamericano de Ciências, Tecnologia, Innovacion y Educacion - ISBN: 978 - 84 - 7666 - 210 - 6 - Artículo 1346, 2014, Buenos Aires - Argentina

BRASIL. ParâmetrosCurriculares Nacionais- Secretaria de Educação Fundamental. Brasília: MEC/SEF, 1997.

BRASIL. Lei de Diretrizes e Bases da Educação Nacional. Lei número 9394, 20 de dezembro de 1996.

CARDOSO, V. C.; OLIVEIRA, S.R. de; KATO, L. A. Percepção de professores sobre o uso de jogos digitais educativos em aulas de matemática. In:Encontro nacional de Educação Matemática, 11., 2013, Curitiba. Anais... Curitiba: SBEM, 2013. p. 1-13.

CAFARTO, R.; VIEIRA, V. Mais da Metade dos alunos de 14 a 17 anos tem nota insuficiente em Português e Matemática. O Estado de São Paulo, São Paulo. 30 Ago. 2018. Disponível em:https://educacao.estadao.com.br/noticias/geral. Acesso em: 2 set.2018.

CARDOSO, T. da S. G. Uso da estimulação transcraniana de corrente contínua em crianças e adolescentes com paralisia cerebral: Efeitos sobre a cognição numérica. 2017. 111f. Tese (Doutorado em Educação e Saúde na Infầncia e Adolescência) Universidade Federal de São Paulo - Guarulhos, SP. 2017.

COELHO NETO, J; ALTOÉ, A. Tecnologias da Informação e Comunicação na Formação de Professores. In: Seminário de Pesquisa do Programa de Pós-Graduação em Educação, 2008, Maringá.

COELHO NETO, João; ALTOÉ, Anair. Construcionismo e a formação de professores: um estudo com alunos do curso de pedagogia da UENP CP. In: Congresso Nacional de Educação - EDUCERE, 10., Curitiba. Anais... Curitiba: PUC, 2011. p. 2316-2325

CORSO, L. V.; DORNELES, B. V. Senso Numérico e Dificuldades de Aprendizagem na Matemática. Revista de Psicopedagogia, Rio Grande do Sul, v. 27, n. 33, p.298309, jan. 2010 .

COSENZA, R. M. GUERRA, L. B; Neurociência e Educação. Como o cérebro aprende. Artimed. Porto Alegre, 2011.

COSTA, A. J. Implicações do Polimorfismos VAL158MET Da Catecol-OMetiltransferase em diferentes aspectos da cognição numérica. $201460 \mathrm{f}$. Dissertação (Mestrado em Neurociências) Universidade Federal de Minas Gerais, Belo Horizonte, MG. 2014

CORSO, L. V.; DORNELES, B. V.. SensoNumérico e Dificuldades de Aprendizagem na Matemática. Revista de Psicopedagogia, Rio Grande do Sul, v. 27, n. 33, p.298-309, maio/ago.2010. 
DEHAENE, S.The Number Sense: How the mind creates Mathematics. Oxford University Press. 1997.

FERNANDES, S. S. As concepções de alunos e professores sobre a utilização de recursos tecnológicos no ensino da Matemática. Monografia. Escola Superior Aberta do Brasil, Vila Velha, 2011.

FERRARI, A. H. O senso numérico da criança: formação e características. 2008. 199 f. Tese (Doutorado em Educação Matemática) Universidade Católica de São Paulo - São Paulo, SP. 2008.

FAJARDO, V. FOREQUE, F. 7 de cada 10 alunos do Ensino Médio têm nível insuficiente em Português e Matemática, diz MEC. em: https:/g1.globo.com/educacao/noticia/2018/08/30/7-de-cada-10-alunos-do-ensinomedio-tem-nivel-insuficiente-em-portugues-e-matematica-diz-mec.ghtml. Acesso em: 02 de Fev. 2018.

GEARY, D. C. Fom infancy to adulthood: the development of numerical abilities. Europe Child \& Adolescent Psychiatry, Columbia, v. 1, n. 9, p.11-16, jan. 2000.

GEARY, D. C. Mathematics and Learning Disabilities. Journal of Learning Disabilities, 37(1), 4-15 (2004).

GENEROSO, A. A; COELHO NETO, J.; REINEHR, S.; MALUCELLI, A. Abordagem Qualitativa do uso das TDIC na Educação Básica. In: CONGRESSO BRASILEIRO DE INFORMÁTICA NA EDUCAÇÃO, 2., 2013, Campinas. Anais... Campinas: UNICAMP, 2013. p. 230-233

GERHARD, T. E.; SILVEIRA, D. T. Método de Pesquisa. Coordenado pela Universidade Aberta do Brasil - UAB/UFRGS e pelo curso de Graduação Tecnológica Planejamento e Gestão para o Desenvolvimento Rural da SEAD/UFRGS - Porto Alegre: Editora da UFRGS, 2009, p. 43-64.

HIGUCHI, A.A. de S. Tecnologias móveis na educação. 2011. 90 f. Dissertação (Mestrado em Educação, Arte e História da Cultura) Universidade Presbiteriana Mackenzie, São Paulo - SP. 2011.

IBCT. Instituto Brasileiro de Informação em Ciências e Tecnologia. Biblioteca Digital Brasileira de Teses e Dissertações. Disponível em: http://bdtd.ibict.br/vufind/. Acesso em :3 jul. 2018.

KITCHENHAM, B. A. Procedures for Performing Systematic Reviews. Tech. Report TR/SE-0401, Keele University, 2014

LARA, I. C. M. Ensino inadequado de Matemática. Revista Ciências e Letras, n. 35, p. 137-152, mar./jul. 2004.

LORENA, A. B. de; CASTRO-CONEGUIM, J. de F.; CARMO, J. dos S. Habilidades numéricas básicas: Algumas contribuições da análise do comportamento. Estudos de Psicologia, São Carlos: v. 3, n. 18, p.439-446, jul. 2013. 
MAIA, D. L.; BARRETO, M. C. Ensinar Matemática com o uso de tecnologias digitais: uma análise a partir da representação social de estudantes de Pedagogia. Revista Educação e Cultura Contemporânea,UFRN,V.11 no 242014, p.136-163.

MARCELINO, C. V. O Viés da Cognição Numérica e Práticas de Controladoria: um estudo quase? Experimental. 2011. 135 f. Dissertação (Mestrado em CONTABILIDADE) Universidade Federal da Bahia, Salvador - BA. 2011.

MARCILESE, M. Sobre o papel da língua no desenvolvimento de habilidades cognitivas superiores: representação, recursividade e cognição numérica. 2011. 111f. Tese (Doutorado em Letras) Pontifícia Universidade Católica do Rio de Janeiro - Rio de Janeiro, RJ, 2011.

MCCLOSKEY. M., CARAMAZZA, A., BASILI, A. Cognitive Mechanism in Number Processing and Calculation: Evidence from Dyscalculia. Brain and Cognition, v. 4, p. 171 $-196,1985$.

MOLINA, J.; RIBEIRO, F.S.; SANTOS, F.H. ASTER, M.V. Cognição Numérica de crianças pré-escolares brasileiras pela ZAREKI-K. Temas em Psicologia, Bauru, v. 23, n. 1, p. 123-135, 2015.

RAMOS, A. C. M.; GOODWIN, F. C.; LAURADES, J. B. A Importância do Senso Numérico na Aprendizagem Matemática.In:VII Encontro Mineiro de Educação Matemática. Universidade Federal de Juiz de Fora. 2015. Minas Gerais.

RAMOS, D. K. A formação de professores para o uso das tecnologias: um mosaico de concepções e emoções. Renote: Novas Tecnologias na Educação, v. 7, n. 1, p.1-11, jul. 2009.

RIBEIRO, F. S. O efeito do treino musical sobre a capacidade da memória operacional e da cognição numérica de crianças com discalculia do desenvolvimento. 2013. 142 f. Dissertação (Mestrado em Psicologia do Desenvolvimento e Aprendizagem) Universidade Estadual Paulista "Júlio de Mesquita Filho" - Bauru, SP. 2013.

RODRIGUEZ, I. A. Treino Musical como proposta para a estimulação da cognição numérica em crianças de idade escolar. 2015115 f. Dissertação (Mestrado em psicologia do Desenvolvimento e Aprendizagem) Universidade Estadual Paulista "Júlio de Mesquita Filho", Bauru - SP. 2015.

SANCHEZ JÚNIOR, S. L. Ensino da Matemática na Educação Infantil e o Desenvolvimento da Cognição Numérica. 2018. 153 f. Dissertação (Mestrado Profissional em Ensino) - Universidade Estadual do Norte do Paraná, Cornélio Procópio, . 2018.

SANTOS, F. H. dos; RIBEIRO, F. S; SILVA, A. da S.; KIKUCHI, R.S.; MOLINA, J.; TONOLI, M.C.Cognição Numérica: Contribuições à Pesquisa Clínica. In: PRADO, P. S. T. do, CARMO, J. dos S. (Org.). Diálogos sobre ensino-aprendizagem da matemática. 
Abordagens pedagógica e neuropsicológica. São Paulo. Cultura Acadêmica, p .63-91, 2016.

SANTOS, F.L. Dos. Discalculia Do Desenvolvimento: Coleção Neuropsicologia na Prática Clínica. São Paulo: Pearson Clinical Brasil, 2017.

SILVA, E. R. DA. Os Efeitos do treino musical sobre a cognição numérica e a memória operacional: um estudo prospectivo em crianças pré - escolares. 2016. 143. f. Dissertação (Mestrado em Psicologia do Desenvolvimento e Aprendizagem) Universidade Estadual Paulista "Júlio de Mesquita Filho", Bauru - SP. 2016.

TAROUCO, L.M.R; et al. Jogos Educacionais. RENOTE: Revista Novas Tecnologias na Educação. UFRGS, V. 2 No 1, Março, 2004

TEIXEIRA, C. F. P.; COELHO NETO, J. O uso das tecnologias digitais para o ensino de matemática financeira: uma revisão sistemática de literatura. Revista Novas Tecnologias na Educação, Rio Grande do Sul, v. 14, n. 2, p.1-10, dez. 2016. Disponível em: https://seer.ufrgs.br/index.php/renote/article/view/70673. Acesso em: 04 jan. 2019.

VALENTE, J.A. Liberando a Mente: computadores na Educação Especial. Campinas: Unicamp, $1991 . \quad 112$ p. Disponível em: https://www.nied.unicamp.br/sites/default/files/livros/livro-liberando-a-mente.pdf Acesso em: 06 Jan 2019. 